\title{
Novel approach to direct adaptive fuzzy control applied to the asynchronous machine
}

\author{
M. Fodil, S. Barkat ${ }^{2}$, B. D. Boukhetala ${ }^{2}$ \\ ${ }^{1}$ Université de M'sila, BP 166, rue ichbillia, M'sila, \\ ${ }^{2}$ Laboratoire de Commande des Processus, Ecole Nationale Polytechnique, BP.152, El-Harach, \\ Alger, \\ E-mail address: dboukhetala@yahoo.fr
}

Keywords: Asynchronous machine, Fuzzy systems, Adaptive control, Fuzzy adaptive law, Stability analysis, Lyapunov function.

\begin{abstract}
This paper presents an advanced direct adaptive fuzzy control for asynchronous machine which uses the theory of approximation and the theory of Lyapunov to establish a parametric adaptation law ensuring the stability and boundedness of all the control signals and the tracking error. In the direct approach, the fuzzy system is used to describe directly the control law and the parameters of the fuzzy system are directly adjusted to achieve the control objectives.

Through the obtained results, stable direct adaptive fuzzy control generalized has proved a great effectiveness and a strong robustness in the presence of parameter variations and disturbances.
\end{abstract}

\section{INTRODUCTION}

The pragmatic use of scientific developments has led to the appearance of modern electric variable speed drive industrial processes. Its optimal operation and its control characteristics directly influence the cost and quality of production.

In this context industrial currently are opting for static converter -asynchronous motor squirrel cage sets because of there robustness, there low cost, and also for the availability of static converters with very high switching frequencies and very sophisticated signal processing means. These technologies allow the implantation of enough complex control strategies.

Nowadays, a large number of publications were dedicated to the problem of nonlinear systems control. The control of complex systems runs up against several difficulties such us the presence of nonlinearities, uncertainties and the computational complexity. This requires intelligent controllers with large capacity for learning and decision making in uncertain environments. Since conventional regulators require a perfect knowledge of the system model to deal with, which does not give a great performance, especially in the presence of modeling errors and / or parametric variations of the system.

In literature, the most cited commands are: vector control by orientation flow [1], input-output linearization control [2], the direct torque control [3], the sliding mode control [4] - [5] and that the fuzzy logic control [6] - [7].

The fuzzy logic control find significant interest from researchers, because the fuzzy systems have the ability to deal with fuzzy variables and induce a control law based on approximate reasoning, as an approximate reasoning we refer to a type of reasoning that is not very accurate or very inaccurate [8] - [9]. This type of system is very useful when the process control presents some uncertainties or unknown variables.

However, to keep a consistent performance in the presence of true uncertainties, the use of adaptive control is in most cases inevitable. Adaptive fuzzy control has been the subject of intensive research during the last decade [10] -[11] - [12] - [13] - [14] - [15] - [16]. 
Therefore, the combination of adaptivity and uncertainty has enabled researchers to derive adaptive fuzzy controllers.

In most work on fuzzy control of the asynchronous machine, the control laws are fixed parameters.

These control techniques have shown their limits during parametric variations of the system. To overcome this drawback, we propose to work with an approach to adaptive control based on fuzzy systems.

There are two approaches to adaptive fuzzy control which are indirect and direct adaptive fuzzy control [17] - [18] -[19] - [20] - [21]. The application of the indirect approach on the asynchronous machine does not guarantee the convergence of the tracking error to zero and the control calculation is performed in two stages, which requires a relatively high [7] computation time.

To overcome these drawbacks, we propose a stable direct adaptive fuzzy generalized control; this technique uses the theory of approximation and the theory of Lyapunov to establish a parametric adaptation law ensuring the stability and boundedness of all the control signals and the tracking error.

In the direct approach, the fuzzy system is used to describe directly the control law and the parameters of the fuzzy system are directly adjusted to achieve the control objectives.

\section{STABLE DIRECT ADAPTIVE FUZZY CONTROLGENERALIZED}

There are several types of direct adaptive fuzzy controller. Among them the adaptive fuzzy control linearized or application the direct control strategy requires the measurement of the derivative of flow and of speed [7]. To overcome this problem, we propose a new type of control namely the stable direct adaptive fuzzy control generalized or the control law is based on Lyapunov stability theory and the theory of approximation by fuzzy systems.

\section{Structure of the control}

Consider a system with a dynamic model of the controller is described by the following equation:

$$
u_{i}=F(X) x_{i}^{n)}+G_{i}(X)
$$

Our objective is to generate a control law for the output to be able to follow its reference $y_{d}$.

For this, we assume that the derivative of the function F (X) satisfies the following condition [20] [21].

$$
\left\|F_{i}(X)\right\|<F_{0}\|X\| \forall X \in \Omega_{c} \text { with } F_{0 i} \square \mathbf{O}
$$

where $\Omega_{c} \subseteq \mathfrak{R}^{n \times n}$ subspace is a state where in the path may vary as a result of the control $F_{0 i}$ is a positive constant and known.

To synthesize the control law we assume that the nonlinear functions $F(X)$ and $G_{i}(X)$ are replaced by fuzzy systems of Takagi-order, based on the M-rules.

$$
F(X)=W_{f i}(X) \theta_{f i}+\varepsilon_{f i}
$$

$$
G_{i}(X)=W_{g i}(X)_{g i}+\varepsilon_{g i}
$$


where $\theta_{f i}, \theta_{g i}$ are the optimal parameters and $\varepsilon_{f}, \varepsilon_{g}$ are errors reconstructions of functions $F(X)$ and $G_{i}(X)$ such that:

$$
\begin{gathered}
\varepsilon_{f i} \leq \varepsilon_{f i} \\
\varepsilon_{g i} \leq \varepsilon_{g i}
\end{gathered}
$$

According to the fuzzy system parameters used $W, \theta_{f i}$ and $\theta_{g i}$ are organized as follows:

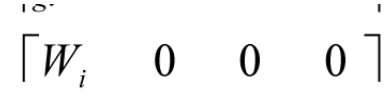

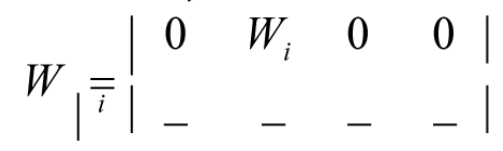

$$
\begin{aligned}
& \left\lfloor\begin{array}{llll}
0 & 0 & 0 & W_{i}
\end{array}\right\rfloor \\
& \theta_{f i}=\left[\begin{array}{ccccc}
\theta_{f i}(1,1) & \theta_{f i}(1,2) & - & - & \theta_{f i}(1, n) \\
\theta_{f i}(2,1) & \theta_{f i}(2,2) & - & - & \theta_{f i}(2, n) \\
- & - & - & - & -
\end{array} \mid\right. \\
& \theta_{g i}=\left[\begin{array}{ccccc}
\theta_{f i}(n, 1) & \theta_{f i}(n, 2) & - & - \\
{ }_{g 1} & \theta_{g 2}^{T} & - & - & \theta_{g n}^{T}
\end{array}\right]^{T} \\
& \theta_{f i}(k, j)=a_{k j}^{1} \quad a_{k j}^{2} \quad-\quad-a_{k j}^{M}, \mathrm{k}, \mathrm{j}=1, \ldots, \mathrm{n}
\end{aligned}
$$

$$
\begin{aligned}
& \theta_{g k}=\left[\begin{array}{llllll}
a_{1}^{G k} & a_{2}^{G k} & - & - & - & a_{M}^{G k}
\end{array}\right] \\
& W(x)=\left[\frac{\alpha_{1}}{\sum_{K=1}^{M} \alpha_{K}} \frac{\alpha_{2}}{\sum_{K=1}^{M} \alpha_{K}}--\frac{\alpha_{M}}{\sum_{K=1}^{M} \alpha_{K}}\right] \\
& \alpha_{K}=\prod_{i=1}^{n} \mu_{A i l i}\left(x_{i}\right) \quad \text { for } l_{i} \in\left\{1, \ldots, m_{i}\right\} \text { et } \\
& K=\{1, \ldots, M\}
\end{aligned}
$$

\section{Control synthesis}

In this work, our goal is to develop a fuzzy adaptive control law for the direct dynamic system represented by (1). We first determine the dynamics of the error filtered according to the fuzzy model, the desired trajectory and the input vector. Then the Lyapunov approach is used to ensure the convergence of the filtered error, the boundedness of adaptive parameters and all control signals $[20]-[21]$. 
Present the error filtered by:

$$
\begin{aligned}
& S_{i}=\left(\frac{}{\partial t}+\lambda_{i}\right)^{(n-1)} \cdot e_{i} \quad \lambda_{i} \square 0 \\
& S=\left[S_{1}, S_{2}, \ldots, S_{n}\right]^{T} \\
& e_{i}=x_{d i}-x_{i} \quad \mathrm{i}=1, \ldots, \mathrm{n} .
\end{aligned}
$$

Obtained

$$
\begin{aligned}
& S_{i}=\lambda_{i}^{(n-1)} \cdot e_{i} i+(n-1) \lambda_{i}^{(n-2)} \cdot \dot{e}_{i}+\ldots \ldots \\
& +(n-1) \lambda_{i} \cdot e^{(n-2)}+e^{(n-1)}
\end{aligned}
$$

The relation (12) can be written in the following compact form:

$S_{i}=C_{i}^{T} \cdot Y_{i}$

with $Y_{i}=\left[\begin{array}{lllll}e_{i} & e_{i} & \ldots & e_{i}^{(n-2)} & e_{i}^{(n-1)}\end{array}\right]^{T}$

$C_{i}^{T}=\left[\begin{array}{lllll}\lambda_{i}^{(n-1)} & (n-1) \lambda_{i}^{(n-2)} & \ldots & (n-1) \lambda_{i} & 1\end{array}\right]$

The dynamics of the filtered error is given by

$S_{i}=C_{r i}^{T} Y_{i}+e_{i}^{(n)}$

whiere

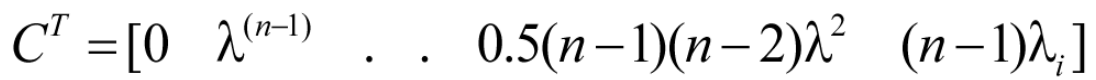

So the dynamics of $\mathrm{S}$ can be written as follows

$S=C_{r}^{T} Y+e^{(n)}$

According to the expression (1) we can write

$x_{i}^{(n)}=F_{i}^{-1}(X)\left[u_{i}(t)-G_{i}(X)\right]$

$e_{i}^{(n)}=x_{d i}^{(n)}-x_{i}^{(n)}=x_{d i}^{(n)}-F_{i}^{-1}(X)\left[u_{i}(t)-G_{i}(X)\right]$

Substituting equation (17) into (15) we obtain:

$S_{i}=C_{r i}^{T} Y_{i}+x_{d i}^{(n)}-F_{i}^{-1}(X) u_{i}(t)-G_{i}(X)$

Note that the filtered reference is given by

$y_{r e f i}=x_{d i}^{(n)}+C_{r i}^{T} Y_{i}$ 


$$
S_{i}=y_{\text {refi }}-F_{i}^{-1}(X)\left[u_{i}(t)-G_{i}(X)\right]
$$

This is equivalent to

$$
F_{i}(X) \bar{S}_{i}=F_{i}(X) y_{\text {refi }}-u_{i}(t)+G_{i}(X)
$$

Replace functions $F_{i}(X)$ and $G_{i}(X)$ by fuzzy systems presented above, the dynamics of the filtered error can take the final form:

$$
F_{i}(X) S_{i}=W_{f i}^{T} \theta_{f i} y_{r e f i}+W_{g i}^{T} \theta_{g i}+\varepsilon_{f i}(t) y_{r e f i}+\varepsilon_{g i}(t)-u_{i}(t)
$$

That the tracking error converges asymptotically to zero, the command will be given by:

$$
u_{i}(t)=K_{d i} S_{i}+\frac{1}{2} F_{0 i}\|X\| S_{i}+W_{f i} \hat{\theta}_{f i} y_{r e f i}^{(n)}+W_{g i} \hat{\theta}_{g i}+K_{i} \operatorname{sigh}\left(S_{i}\right)
$$

Where $K_{i}$ is the term of the sliding mode given by:

$$
K_{i}=\bar{\varepsilon}_{f i}\left\|y_{r e f i}\right\|+\bar{\varepsilon}_{g i}
$$

Where the parameter vectors $\hat{\theta}_{f i} e t \hat{\theta}_{g i}$ are adapted by

$\hat{\theta}_{f i}=\gamma_{1 i} W_{f i}(X) S_{i}\left(Y_{r e f i}^{(n)}\right)^{T}$

$\hat{\theta}_{g i}=\gamma_{2 i} W_{g i}(X) S_{i}$

And parameters $F_{i}^{0},{ }^{\varepsilon}, f_{i}, \quad g i$ are adjusted by adaptation laws given below

$$
\begin{aligned}
& \hat{F}_{0 i}=\eta_{i}\left\|X \left|\left\|\mid S_{i}\right\|\right.\right. \\
& \overrightarrow{\varepsilon_{f i}}=\eta_{i}\left\|Y_{r e f i}^{(n)}\right\|\left\|S_{i}\right\| \\
& \bar{\varepsilon}_{g i}=\eta_{i}\left\|S_{i}\right\|
\end{aligned}
$$

Where $\gamma_{1 i}, \gamma_{2 i}, \eta_{i \square}>0 \quad$ for $\mathrm{i}=1,2$. 
The block diagram of the stable direct adaptive fuzzy control generalized is illustrated in Figure (1).

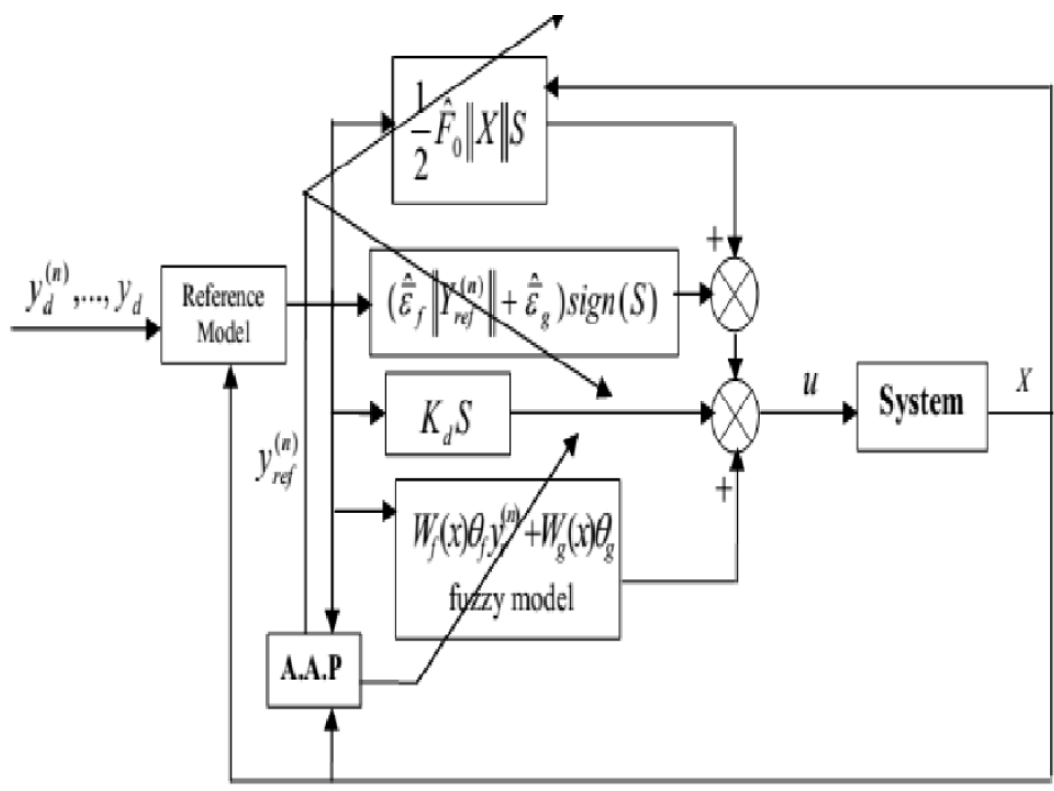

Fig (1) : Structure of the stable direct adaptive fuzzy control generalized.

\section{STUDY OF THE STABILITY}

Consider the system (1) controlled by the adaptive law given by

$u_{i}(t)=K_{d i} S_{i}+\frac{1}{2} \hat{F}_{0 i}\|X\| S_{i}+W_{f i} \hat{\theta}_{f i} Y_{r e f i}^{(n)}+W_{g i} \hat{\theta}_{g i}$

$+\left(\hat{\bar{\varepsilon}}_{f i}\left\|Y_{r e f i}^{(n)}\right\|+\hat{\bar{\varepsilon}}_{g i}\right) \operatorname{sign}\left(S_{i}\right)$

Where $\hat{F}_{0 i}, \hat{\bar{\varepsilon}}_{f i}, \hat{\bar{\varepsilon}}_{g i}$ are unknown parameters.

In order to demonstrate the stability of the closed loop system, we choose the following Lyapunov function:

$V_{i}=\frac{1}{2} S_{i}^{T} F_{i}(X) S_{i}+\frac{1}{2 \gamma_{1}} \tilde{\theta}_{g i}^{T} \tilde{\theta}_{g i}+\frac{1}{2 \gamma_{2}} \tilde{\theta}_{f i}^{T} \tilde{\theta}_{f i}$

$+\frac{1}{2 \eta^{i}}\left(\widetilde{F}^{0 i}\right)^{2}+\frac{1}{2 \eta^{i}}\left(\bar{\varepsilon}^{f i}\right)^{2}+\frac{1}{2 \eta^{i}}\left(\underline{\xi}_{g i}\right)^{2}$

$E_{0 i}^{2 \eta}=F_{0 i}-\hat{F}_{0 i} \quad \bar{\varepsilon}_{f i}=\bar{\varepsilon}_{f i}-\hat{\bar{\varepsilon}}_{f i}^{2 \eta} \quad \bar{\varepsilon}_{g i}=\bar{\varepsilon}_{g i}-\hat{\bar{\varepsilon}}_{g i}$

After derivation of the function $V_{i}$, we obtain $V_{i}=\frac{1}{S_{i}^{T}} F_{i}(X)+S_{i}^{T} F_{i}(X) S_{i}-\frac{1}{\tilde{\theta}_{g i}^{T}} \hat{\theta}_{g i}$ 
After derivation of the function $V_{i}$, we obtain

$$
\begin{aligned}
& V_{i}=\frac{1}{-} S_{i}^{T} F_{i}(X)+S_{i}^{T} F_{i}(X) S_{i}-\frac{1}{-\tilde{\theta}_{g i}^{T} \hat{\theta}}{ }_{g i} \\
& -\frac{1}{2} \tilde{\theta}^{T} \hat{\theta}-\frac{1}{\tilde{F} \hat{F}}-\frac{1}{\widetilde{\varepsilon}} \widetilde{\hat{\varepsilon}^{\gamma}}-\frac{1}{\gamma^{1 i}} \widetilde{\bar{\varepsilon}} \hat{\bar{\varepsilon}}
\end{aligned}
$$

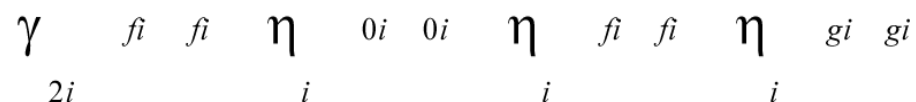

Replace $F(X) S$ given by the expression (22) in this last, we obtain:

$$
\begin{aligned}
& V_{i}=\mathcal{Z} S_{i}^{T} F_{i}(X)+S_{i}^{T}\left[W_{f i}^{T} \theta_{f i} Y_{r e f i}+W_{g i}^{T} \theta_{g i}\right. \\
& \left.+\varepsilon_{f i} Y_{r e f i}+\varepsilon_{g i}-u_{i}(t)\right]-\frac{1}{\gamma_{1 i}} \tilde{\theta}_{g i}^{T} \hat{\theta}_{g i}^{\hat{T}}-\frac{1}{\gamma_{2 i}} \tilde{\theta}_{f i}^{T} \hat{\theta}_{f i}
\end{aligned}
$$

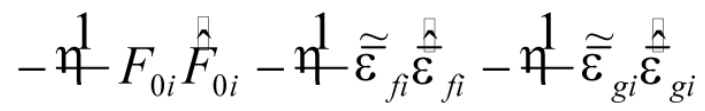

$$
\begin{aligned}
& V^{V}=V_{1}+V_{2}+V_{3} \\
& \text { For } V_{1}=-K_{d i} S_{i}^{T} S_{i} \\
& V_{2}=\frac{1}{2} S_{i}^{T} \hat{F}(X)-\frac{1}{2} S_{i}^{T} \hat{F}_{0 i}\|X\| S_{i}-\frac{1}{\eta_{i}} \widetilde{F}_{0 i} \hat{F}_{0 i} \\
& V_{3}=S_{i}^{T}\left(\varepsilon_{f i} Y_{r e f i}+\varepsilon_{g i}\right)-S_{i}^{T}\left(\hat{\bar{\varepsilon}}_{f i}\left\|Y_{r e f i}\right\|+\hat{\bar{\varepsilon}}_{g i}\right) \operatorname{sign}\left(S_{i}\right) \\
& -\frac{1}{\eta_{i}} \widetilde{\widetilde{\varepsilon}_{f i}} \hat{\bar{\varepsilon}}_{f i}-\frac{1}{\eta_{i}} \widetilde{\bar{\varepsilon}_{g i}} \hat{\bar{\varepsilon}}_{g i}
\end{aligned}
$$

Knowing that $K_{d i}$ is a positive constant, it comes

$$
\bar{V}_{1} \leq 0
$$

According to the adaptation law (25) we have:

$$
\bar{V}_{2} \leq 0
$$

Using the laws of modifications (26) and (27), it follows:

$$
V_{3} \leq 0
$$

Where

$$
V \leq 0
$$

Therefore, $\mathrm{S}$ asymptotically converges to zero and that all signals in the system are bounded. 


\section{APPLICATION TO THE ASYNCHRONOUS MACHINE}

In the fixed reference relative to the rotating field. The flow dynamics $\phi_{\mathrm{r}}$ and the rotating speed pulsation $\omega_{\mathrm{r}}$ of three-phase asynchronous motor are given by the following equations:

$$
\begin{aligned}
& \frac{d \phi_{d r}}{d t}=\frac{M}{T_{r}} i_{d s}-\frac{\phi_{d r}}{T_{r}}+\left(\omega_{s}-\omega_{r}\right) \phi_{q r} \\
& \frac{d \phi_{q r}}{d t}=\frac{M}{T_{r}} i_{q s}-\frac{\phi_{q r}}{T_{r}}-\left(\omega_{s}-\omega_{r}\right) \phi_{d r} \\
& \frac{d \omega_{r}}{d t}=\frac{P^{2} M}{J L_{r}}\left(\phi_{d r} i_{q s}-\phi_{q r} i_{d s}\right)-\frac{P C_{r}}{J}-\frac{f \omega_{r}}{J}
\end{aligned}
$$

where $\left(i_{d s}, i_{q s}\right)$ are the components of the stator current

$i_{d s}=\frac{T_{r}}{M} \frac{d \phi_{r}}{d t}+\frac{1}{M} \phi_{r}$

$i_{q s}=\frac{J L_{r}}{P^{2} M \phi_{r}} \frac{d \omega_{r}}{d t}+\frac{L_{r} f}{P^{2} M \phi_{r}} \omega_{r}+\frac{L_{r}}{P M \phi_{r}} C_{r}$

with $\phi_{r}$ : flows resulting $\quad \phi_{r}=\sqrt{\phi_{d r}^{2}+\phi_{q r}^{2}}$

where motor parameters are:

$C_{r}:$ The load torque

$\mathrm{J}$ : Moment of inertia

$\mathrm{P}$ : Number of pole pairs

$f$ : Coefficient of friction

$L_{r}, L_{s}:$ Inductance cyclic stator and rotor per phase

$\mathrm{M}$ : Cyclic mutual inductance between stator and rotor

$$
T_{r}=\frac{L_{r}}{R_{r}}: \text { Rotor time constant }
$$

Adaptive fuzzy controllers speed and flow provide currents $i_{d s r e f}$ and $i_{q s r e f}$ providing regulation of $i_{d s}$ and $i_{q s}$ respectively. The reference currents are obtained by the passage of $i_{d s r e f}$ and $i_{q s r e f}$ by a coordinate transformation. Comparison of current references to real currents provides control of each arm of the inverter. By the application of the direct adaptive fuzzy control generalized for regulating the speed of the asynchronous machine, the system is put in the following form: 


$$
\left\{\begin{array}{l}
i_{\text {dsref }}=F_{1}\left(\phi_{r}\right) \phi_{r}+G_{1}\left(\phi_{r}\right) \\
i_{\text {qsref }}=F_{2}(\Omega) \Omega+G_{2}(\Omega)
\end{array}\right.
$$

Where the dynamics of the flow depends only on the control input $i_{d r e f}$ and flow $\phi_{r}$ then, the dynamic of the speed depends mainly on the input control $i_{q r e f}$ and speed $\Omega$.

The implementation of this control requires the approximation of functions $F$ and $G_{i}$ by fuzzy systems:

$$
\begin{aligned}
& \left\{\begin{array}{l}
F_{1}\left(\phi_{r}\right)=W_{f 1}\left(\phi_{r}\right) \theta_{f 1}+\varepsilon_{f 1} \\
G_{1}\left(\phi_{r}\right)=W_{g 1}\left(\phi_{r}\right) \theta_{g 1}+\varepsilon_{g 1}
\end{array}\right. \\
& \left\{\begin{array}{l}
F_{2}(\Omega)=W_{f 2}(\Omega) \theta_{f 2}+\varepsilon_{f 2} \\
G_{2}(\Omega)=W_{g 2}(\Omega) \theta_{g 2}+\varepsilon_{g 2}
\end{array}\right.
\end{aligned}
$$

In this approximation the evaluation functions $F_{i}$ and $G_{i}$ is performed with three fuzzy rules and therefore the flow and the speed are described by three fuzzy sets. Using the latter method, we calculate the input command as follows:

$$
\left\{\begin{array}{l}
i_{d s r e f}=K_{d 1}+0.5 \hat{F}_{01}\left|\varphi_{r}\right| S_{1}+W_{f 1}^{T} \hat{\theta}_{f 1} Y_{r e f 1}+W_{g 1}^{T} \hat{\theta}_{g 1} \\
+\left(\hat{\bar{\varepsilon}}_{f 1}\left|Y_{r e f 1}\right|+\hat{\bar{\varepsilon}}_{g 1}\right) \operatorname{sigh}\left(S_{1}\right) \\
i_{q s r e f}=K_{d 2}+0.5 \hat{F}_{02}|\Omega| S_{2}+W_{f 2}^{T} \hat{\theta}_{f 2} Y_{r e f 2}+W_{g 2}^{T} \hat{\theta}_{g 2} \\
+\left(\hat{\bar{\varepsilon}}_{f 2}\left|Y_{r e f 2}\right|+\hat{\bar{\varepsilon}}_{g 2}\right) \operatorname{sigh}\left(S_{2}\right)
\end{array}\right.
$$




$$
\begin{aligned}
& \frac{\hat{\varepsilon}}{\bar{\varepsilon} 1}=\eta_{1}\left|S_{1}\right| \quad \quad \frac{\hat{\varepsilon}}{g 2}=\eta_{2}\left|S_{2}\right| \\
& \hat{F}_{01}=\eta_{1}\left|\varphi_{r}\right|\left|S_{1}\right| \quad \hat{F}_{02}=\eta_{2}|\Omega|\left|S_{2}\right| \\
& \hat{\theta}_{f 1}=\gamma_{11} W_{f 1} S_{1} Y_{r e f 1} \quad \hat{\theta}_{g 1}=\gamma_{12} W_{g 1} S_{1} \\
& \hat{\theta}_{f 2}=\gamma_{21} W_{f 2} S_{2} Y_{r e f 2} \quad \hat{\theta}_{g 2}=\gamma_{22} W_{g 2} S_{2}
\end{aligned}
$$

With $K_{d 1}, K_{d 2}, \gamma_{11}, \gamma_{12}, \gamma_{21}, \gamma_{22}, \eta_{1}, \eta_{2}$ are positive constant, while $S_{i}$ and $Y_{\text {refi }}$ respectively are the error and the reference signal. His expressions are given by:

$$
\begin{aligned}
& S_{1}=\phi_{r e f}-\phi_{r} \\
& S_{2}=\Omega_{r e f}-\Omega \\
& Y_{r e f 1}=\phi_{r e f}+\lambda_{1}\left(\phi_{r e f}-\phi_{r}\right) \\
& Y_{r e f 2}=\Omega_{r e f}+\lambda_{2}\left(\Omega_{r e f}-\Omega\right)
\end{aligned}
$$

Figure (2) schematically illustrates the principle of the adaptive fuzzy control generalized when setting the asynchronous machine. 


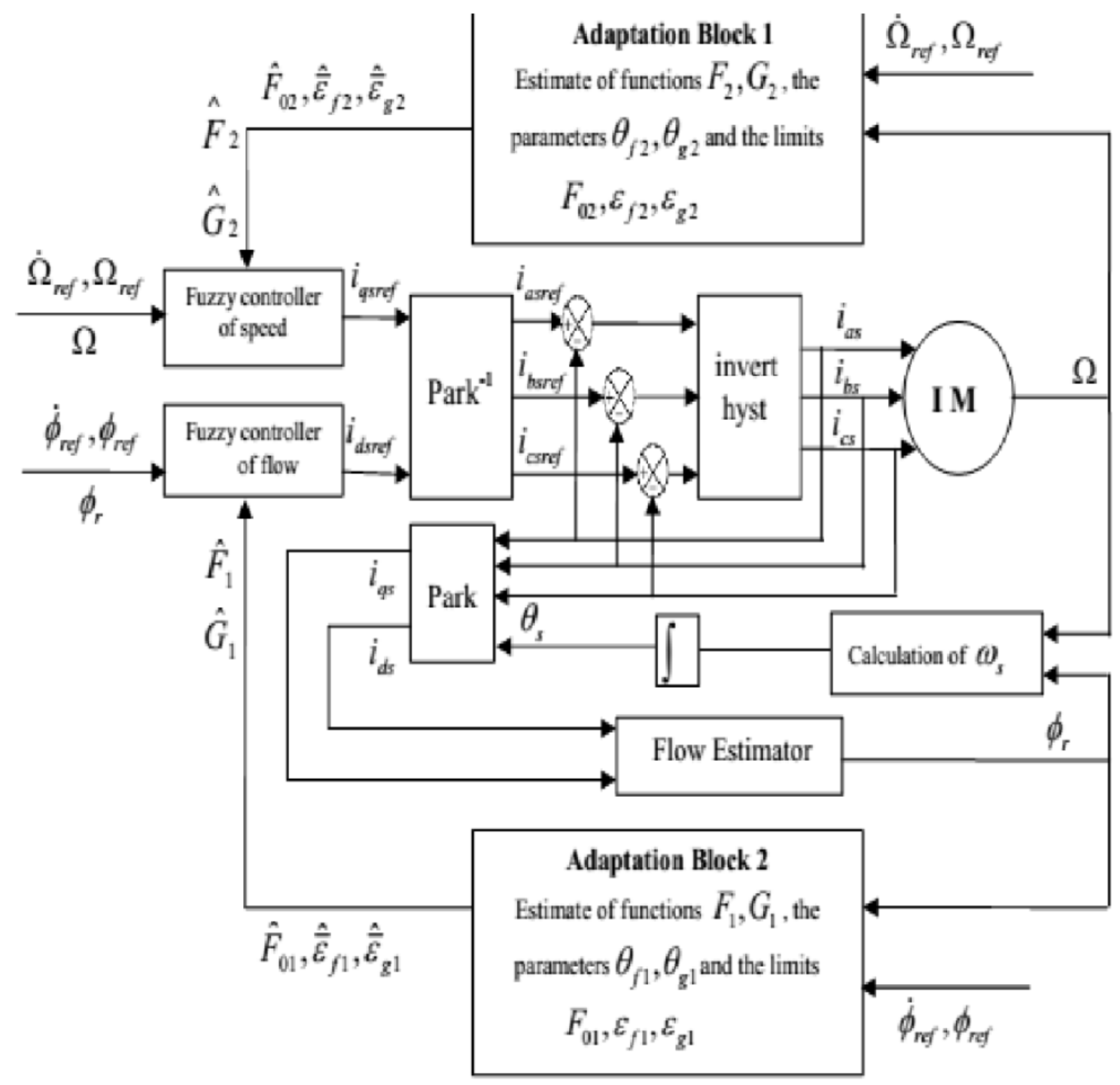

\section{Fig (2) : Structure speed control method by the stable direct adaptive fuzzy control generalized.}

\section{SIMULATION RESULTS}

Given the nature of the "sign" function contained in the term of the sliding mode, the control is discontinuous in the vicinity of the origin $(S=0)$, this can cause nuisance switching (chattering) command. To overcome this problem, we replaced the "sign" function by a continuous "smooth" function:

$\operatorname{smooth}(x)=\frac{x}{|x|+0.5}$

Figures (3), (4) respectively illustrates the responses obtained when starting of the asynchronous machine for a set point speed 209rad / s and load change on reversal of the direction of travel. We can see that the speed perfectly follows its reference, rejection of the disturbance is fast, the stator current is limited during the transitional regime and the rotor flux is maintained, it follows its reference value according to the axis (d) with a quadrature component (q) zero. 

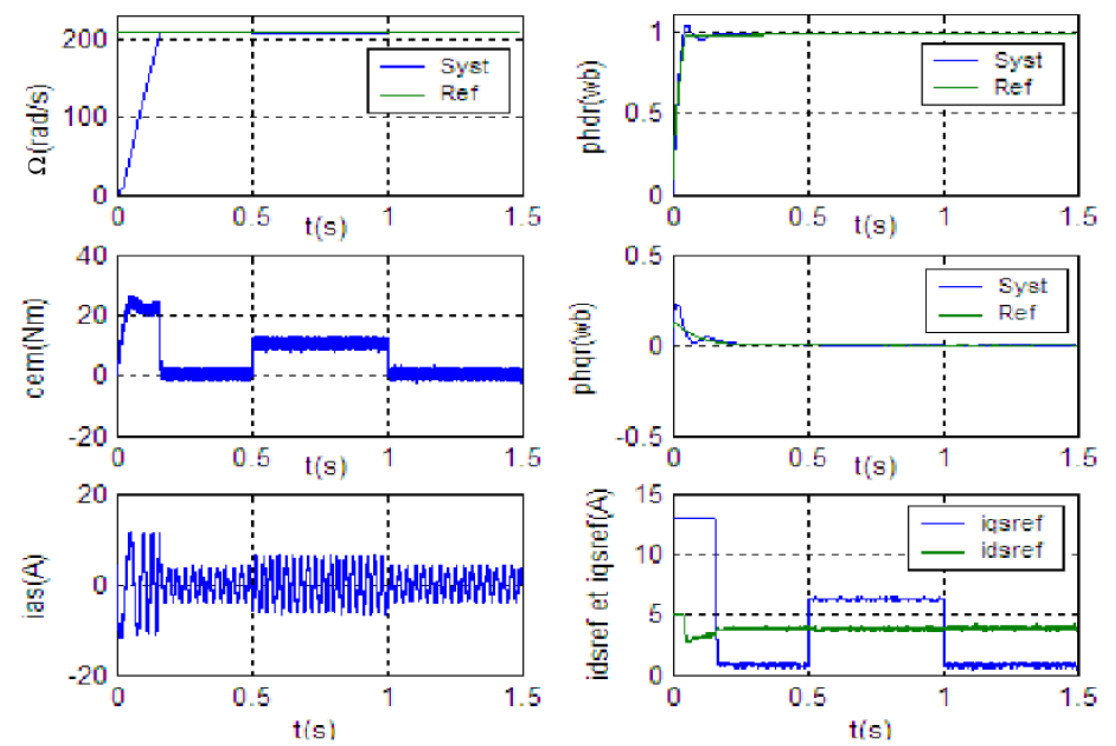

Fig.3: Dynamic behavior of the MAS with load variation.
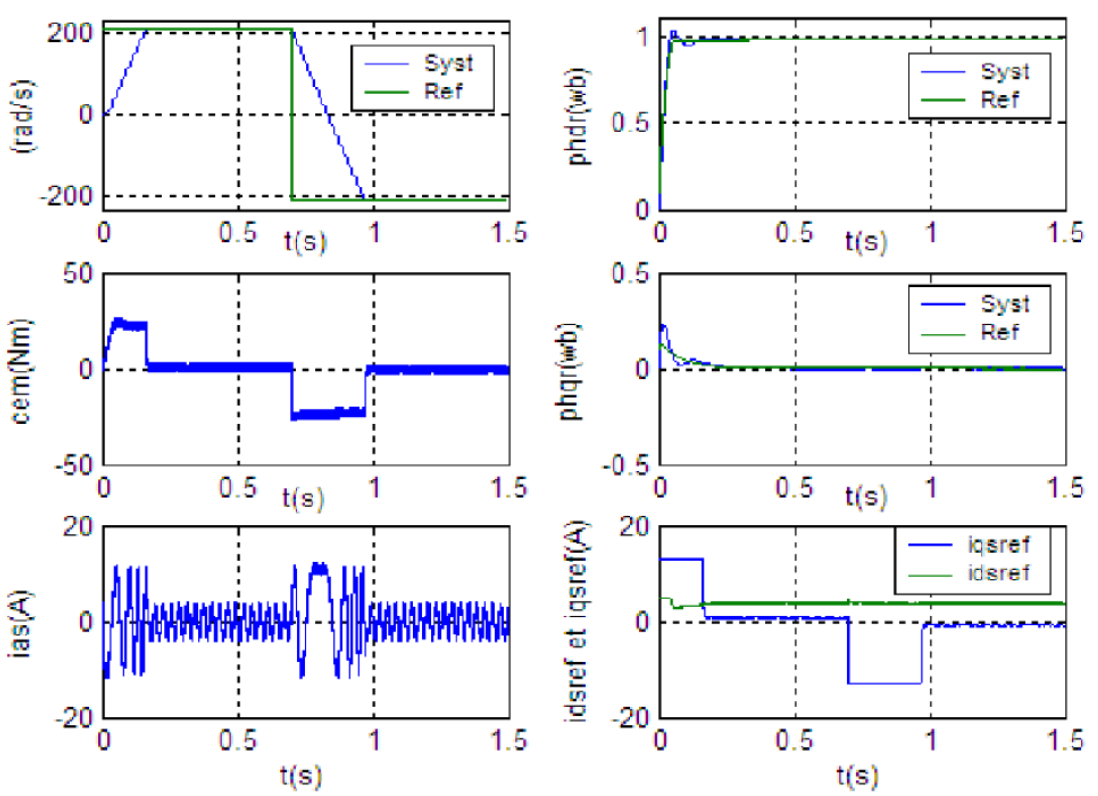

Fig.4: Dynamic behavior of the MAS with reverse-speed.

\section{ROBUSTNESS}

To test the robustness of the generalized fuzzy adaptive control, we made a parametric change on the rotor resistance, on the rotor and stator inductances well as the mutual inductance. We can conclude from the results illustrated in Figure (5), the direct adaptive fuzzy control stability generalized exhibits strong robustness in the presence of parametric variations. Dynamic tracking of the setpoint and the decoupling of the machine are not affected by these changes, which shows the adaptation of the proposed control capacity. 

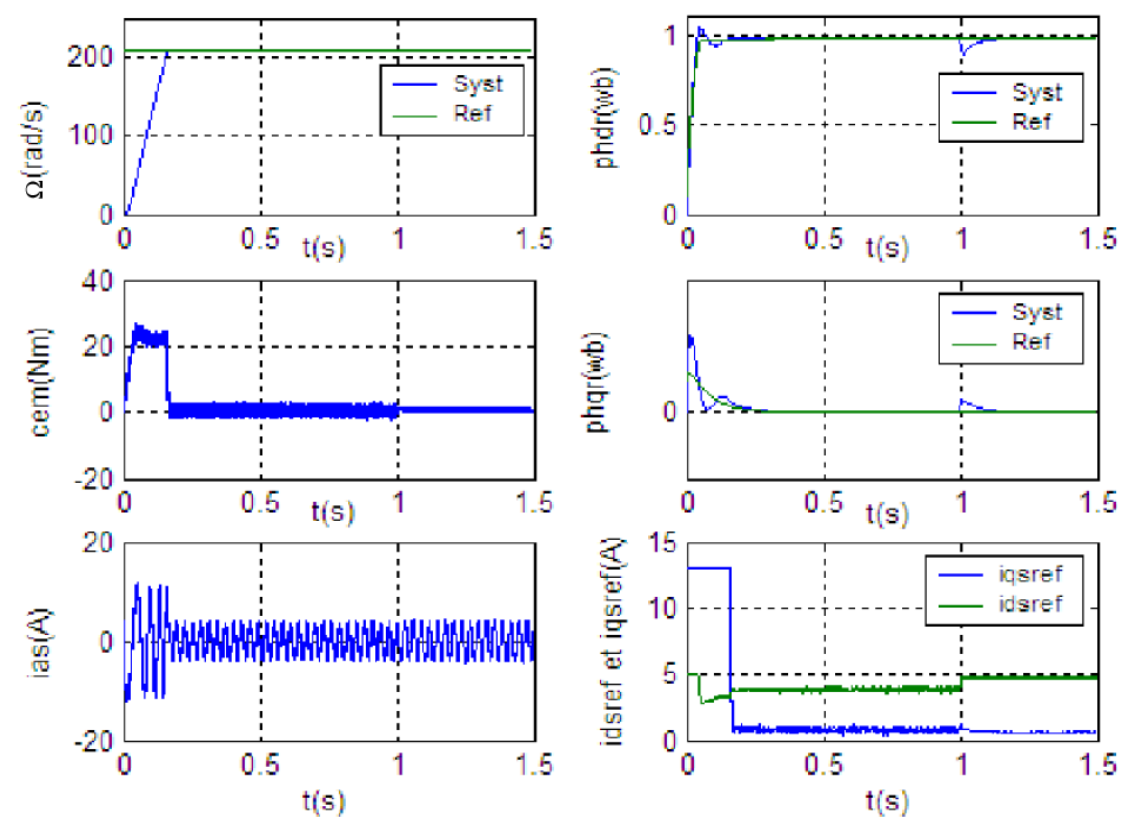

Fig. 5: Dynamic behavior of the MAS when starting with parametric variation

\section{CONCLUSION}

The non-linear characteristic and variation of parameters of the asynchronous machine during operation poses a control problem. The use of modern control methods allows to obtain good performance. This work relates setting the speed of the asynchronous machine powered by an inverter controlled voltage current. Thus we are interested in fuzzy control systems that are capable of treating imprecise, uncertain and vague.

In this case, we presented a stable direct adaptive fuzzy control generalized. or control laws with easy structure to implement and does not require a structural or parametric information of the dynamic model of the asynchronous machine and fuzzy systems are involved to approximate the model of the system to adjust. This control technique using approximation theory and the Lyapunov to establish a law parametric adaptation ensuring boundedness of all system signals and error parameters of fuzzy systems, and to offset the effects of reconstruction errors is introducing a term of sliding mode. From the results obtained, stable direct adaptive fuzzy control generalized has good performance as well as in the presence of disturbance that upon reversal of the direction of rotation. The techniques of direct adaptive fuzzy control generalized is tested by the parametric variations of the system, and the results recorded, have allowed us to determine that this technique exhibits strong robustness in the presence of parametric variations.

\section{References}

[1] J.P.Caron,J.P.Hautier,"Modelisation et commande de la machine asynchrone,"EditionTechnip Paris 1995.

[2] A. Isidori, "Nonlinear Control Systems." 2nd Edition. New York, NY Springer.Verlag, 1989.

[3] J. Carvajal, G. Chen et H. Ogmen, "Fuzzy PID controller: design performance evaluation and stability analysis," Inter. Jour, of Information Sciences, pp. 249.170, 2000.

[4] M. Djemai, J.P. Barbot, A. Glumineau, R. Boisliveau, "Nonlinear Flux Sliding mode Oberver for Induction Motor ." Experimental study. 3rd imaacs/ieee International Multiconférence, Athens, Grèce 1999. 
[5] H. Chekireb, M. Tadjine, M. Djemaï, "On a class of manifolds for sliding mode Control of induction motor". Journal of Nonlinear Dynamics and Systems Theory, pp. 1.14, 2008.

[6] J.Fonseca et al,"Fuzzy logic speed control of induction motor,"microprocessors and Microsystems ,22,pp.523-534,1999.

[7]M.Fodil,S.Barkat,D.Boukhetala,"Commande adaptative par logique floue de la machine asynchrone," thèse de magister, univ M'sila , 2008.

[8] L.X. Wang, J.M. Mendel, "Fuzzy basis functions, universal approximation, and orthogonal least square learning," IEEE Trans. Neural Networks pp.807-814,1992. [9] L.X. Wang, " Adaptive fuzzy systems and control," design and analysis. Prentice-hall, englewood clifs, Nj, 1994.

[10] L.X. Wang, " Adaptive fuzzy control of linear systems," IEEE Trans.on fuzzy systems,1(1),pp. 146-155,1993.

[11] I. K. Hazzab, Bousserhane, M. Kamli \& M. Rahli, "Adaptive fuzzy sliding mode controller for induction motor control," ICTTA'06 IEEE in Conf. on Information and Communication Technologies: from Theory to Applications, vol. 1, pp. 163-168, April 2006. [12] H. N. Nounou H. Rehman, "Application of adaptive fuzzy control to ac machine,"Applied Soft Computing, vol.7, no. 3, pp.899.907, Jun 2007

[13] N. Ezziani," Commande adaptative floue backtepping d'une MAS avec et sans capteur mecanique,"these de doctorat,univ Reims Champagne,2010.

[14] Y.J. Liu, S.C. Tong, T.S. Li, "Observer-based adaptive fuzzy tracking control for a class of uncertain nonlinear MIMO systems", Fuzzy Sets Syst.164, pp.25-44,2011.

[15] A.Boulkroune, M.Saad, M.Farza,"adaptive fuzzy control for a class of MIMo nonaffine uncertain systems,"Neuro Computing 93,pp.48-55,2012.

[16] A. Boulkroune, M. M'saad,"On the design of observer-based fuzzy adaptive controller for nonlinear systems with unknown control gain sign," Fuzzy Sets and Systems 201,pp.71 - 85,2012.

[17] Y.T. Kim, Z.Bien , " Robust indirect adaptative fuzzy control, " proc.IEEE Int.conf.on fuzzy Systems, Seoul, Korea, pp.1293-1298,1999.

[18] Y.W.Cho,Y.H.YEE, M.Park, "An indirect model reference adaptative fuzzy controlfor SISO Takagi-Sugeno model," proc.IEEE Int.conf.on fuzzy Systems, Seoul, Korea,pp. 474-479,1999.

[19] N. Essounbouli, A. Hamzaoui, " Direct and indirect robust adaptive fuzzy controllers for a class of nonlinear systems," International Journal of control, Automation and Systems, vol. 4, no. 2, pp.146.154, 2006. [20] H. Chekireb, M. Tadjine, D. Bouchaffra, "Direct adaptive fuzzy control of linear system class with application," control and intelligent systems. Vol.31,No.2,pp.113121,2003 . 